\title{
Identification of "Malvastrum yellow vein Lahore virus" a proposed new species of begomovirus in association with cotton leaf curl Multan betasatellite infecting green bean (Phaseolus vulgaris) in Pakistan
}

\author{
Saleem Ur Rahman ${ }^{1,2} \cdot$ Muhammad Zubair $^{1,3} \cdot$ Nasim Ahmed $^{1,2} \cdot$ Muhammad Zuhaib Khan $^{1} \cdot \mathrm{Ghulam}^{\text {Raza }}{ }^{1} \cdot$ \\ Imran Amin ${ }^{1}$. Shahid Mansoor ${ }^{1}$
}

Received: 20 August 2019 / Accepted: 6 December 2019 / Published online: 10 December 2019

(C) Australasian Plant Pathology Society Inc. 2019

\begin{abstract}
Begomoviruses; have devastating impact on the yield of many economically important crops around the globe. During field survey in 2016, typical symptoms of begomovirus infections were observed in green beans in district Hazara, Pakistan. We identified a new strain of Malvastrum yellow vein Lahore virus in green beans for the first time in Pakistan.
\end{abstract}

Keywords Begomovirus · Malvastrum yellow vein Lahore virus $\cdot$ Cotton leaf curl Multan betasatellite $\cdot$ Green beans

Geminiviruses consist of twinned icosahedral virion and cause infection in many food crops, weeds and ornamental plants. It is composed of single-stranded DNA (ssDNA) with one or two genomic components (Zerbini et al. 2017). In 2016, during a routine survey of plants for possible virus infections in Hazara district, Khyber Pakhtunkhwa province, green bean (Phaseolus vulgaris) fields were visited. In one field green bean plants were observed with begomovirus-like symptoms consisting of leaf curling, stunted growth, foliar chlorosis and vein thickening (Fig. 1A). The study was designed to characterize begomoviruses in green bean, which can help us to manage and design a broad-spectrum strategy for its control in future.

To check the possible presence of begomovirus in green bean, both symptomatic ( 5 samples) and asymptomatic (2 samples) leaf samples were collected. The genomic DNA of

Saleem Ur Rahman

saleemurrrahman20@gmail.com

Muhammad Zubair

zubair.nibge@gmail.com

Nasim Ahmed

nasimmughal2845@gmail.com

Muhammad Zuhaib Khan

zuhaib_bt@yahoo.com

Ghulam Raza

graza4@gmail.com green beans was extracted using CTAB method (cetyl trimethyl ammonium bromide; Doyle and Doyle 1987). The circular DNA molecules were enriched by rolling circle amplification (RCA) using Ф29 DNA polymerase (Thermo Fisher Scientific, Waltham, MA, USA). The viral components were PCR (polymerase chain reaction) amplified from RCA product using Begomo-F/Begomo-R primer pair for begomoviruses (Shahid et al. 2007) and $\beta 01 / \beta 02$ for betasatellites, respectively (Briddon et al. 2002). For amplification of alphasatellites the primer pair DNA 101/DNA 102 (Bull et al. 2003) was used. The PCR products of $\sim 2.8 \mathrm{~kb}$ and $\sim 1.4 \mathrm{~kb}$ amplified with Begomo-F/Begomo-R and $\beta 01 / \beta 02$ respectively, were purified and cloned in $p T Z 57 R / T$ (Thermo Scientific, USA). No amplification was observed from healthy plant samples. Complete nucleotide sequences of clones were determined by Sanger sequencing using Applied Biosystems

Imran Amin

imranamin1@yahoo.com

Shahid Mansoor

shahidmansoor7@gmail.com

National Institute for Biotechnology and Genetic Engineering (NIBGE), Jhang Road, Faisalabad, Pakistan

2 Pakistan Institute of Engineering and Applied Sciences, Nilore, Islamabad, Pakistan

3 Department of Biotechnology, Hazara University, Mansehra, Pakistan 
Fig. 1 Green bean plant showing symptoms of leaf curling, stunted growth, foliar chlorosis and vein thickening; (A) Healthy green bean plant; (B)

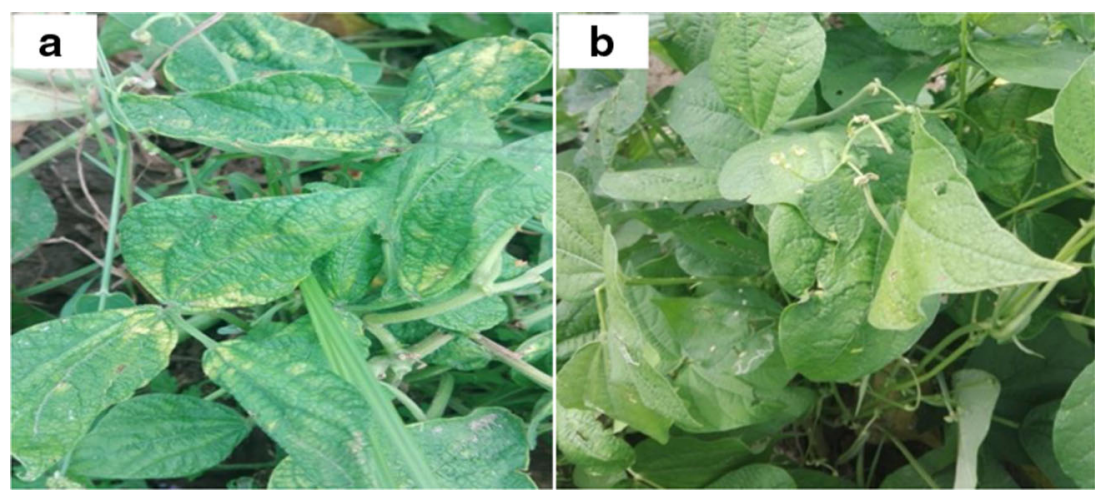

3730XL DNA sequencer. Sequences were analyzed via Lasergene (DNAStar Inc., Madison, USA). Highly similar sequences were searched in the databank (NCBI) using BLASTn search tool. Clone SR-1, obtained from the amplification with Begomo-F/Begomo-R showed maximum identity of $93.2 \%$ with malvastrum yellow vein Lahore virus (MaYVLaV; MF683828), while MaYVLaV (MF683828) itself showed $89.93 \%$ identity with cotton leaf curl Bangalore virus (CLCuBaV; LC316185). The sequence of the clone SR1 was submitted to NCBI and is available under accession number MG551984. The clone SR-51, obtained from the amplification with primer $\beta 01 / \beta 02$, showed $99.8 \%$ identity with cotton leaf curl Multan betasatellite (CLCuMuB; FN432359). The clone SR-51 was also submitted to NCBI and is available under accession number MK098183. In phylogenetic analysis the clone SR-51 clustered with CLCuMuB (FN432359 and HG428707) as shown in fig. 2B. Cotton leaf curl Multan betasatellite is an important species of Betasatellite associated with several monopartite begomoviruses causing Cotton leaf curl disease (CLCuD) that leads to huge economic loss to cotton production in Pakistan (Briddon et al. 2014). There was no amplification from symptomatic green bean plants with alphasatellite-specific primers (results not shown).

The cloned genome of SR-1 was identical to OW monopartite begomoviruses encoding a total of six ORFs (open reading frames). Phylogenetic tree was constructed using maximum likelihood method (with 1000 bootstrap value) in MEGA6 software (Tamura et al. 2013). The SR-1 clustered and formed a branch tree with MaYVLaV (MF683828), identified in Malvastrum coromandelianum in 2013 in Lahore, Pakistan (Fig. 2A). The SDT analysis (Muhire et al. 2014) of SR-1 showed $93.2 \%$ identity with MaYVLaV (MF683828) and $89.2 \%$ identity with hollyhock leaf curl virus (HoLCV; GQ478343). The MaYVLaV (MF683828)

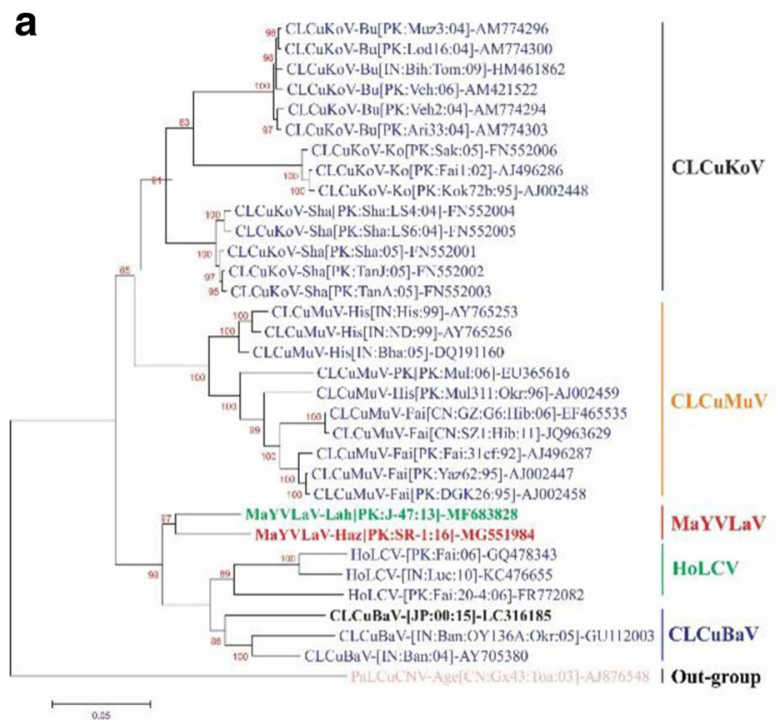

Fig. 2 Maximum likelihood phylogenetic tree constructed on bootstrap method (1000 replications) and rooted on papaya leaf curl China virus (PaLCuCNV; AJ876548). MaYVLaV-Haz (MG551984) is highlighted in red color (bold text) and it clustered with MaYVLaV (MF683828). The accession MaYVLaV-Lah (MF683828) is highlighted green (bold text) and which clustered with cotton leaf curl Bangalore virus (CLCuBaV; b

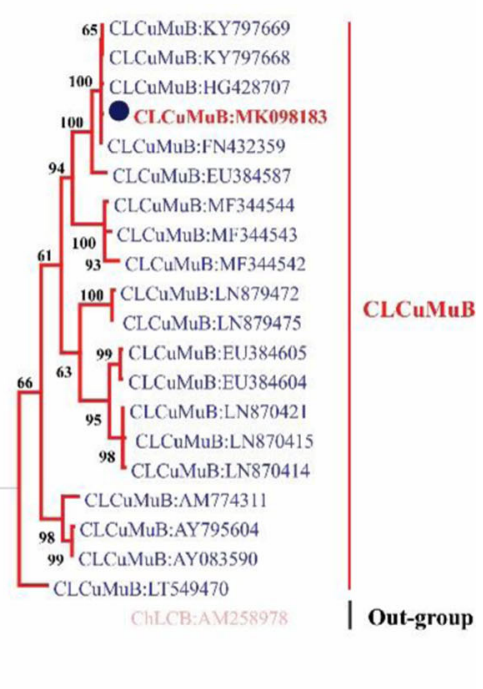

LC316185) as highlighted in black color with bold text; (A). Maximum likelihood phylogenetic tree constructed on bootstrap method (1000 replications) and rooted on chili leaf curl betasatellite (ChLCB; AM258978). The cotton leaf curl Multan betasatellite (CLCuMuB; MK098183) is highlighted in red bold text and clustered with CLCuMuB (HG42707, KY797669 and FN43235); (B) 
sequence showed maximum $89.4 \%$ identity with $\mathrm{CLCuBaV}$ (LC316185), so following the species demarcation criteria for begomovirus (Brown et al. 2015) this sequence was submitted to NCBI with new species named Malvastrum yellow vein Lahore virus. Based on the criteria of strain and species demarcation ( $94 \%$ and $91 \%$ pairwise sequence identity respectively) for begomovirus, SR-1 genome is the novel strain of Malvastrum yellow vein Lahore virus. Here, we propose a name "malvastrum yellow vein Lahore virus-Hazara" (MaYVLaV-Haz) strain to SR-1 sequence. As still paper describing the identification of Malvastrum yellow vein Lahore virus (MaYVLaV) is not published (one sequence (MF683828) is available in NCBI), so here we propose Malvastrum yellow vein Lahore virus (MaYVLaV) a new species of begomoviruses. Now, it has two strains; malvastrum yellow vein Lahore virus-Lahore (MaYVLaVLah; MF683828) and malvastrum yellow vein Lahore virusHazara (MaYVLaV-Haz; MG551984). This is the first report of MaYVLaV-Haz along with CLCuMuB infecting green bean in Pakistan. Previously, few begomoviruses were identified infecting green beans across the globe, such as appearance of Tomato yellow leaf curl virus on green bean in Spain (Navas-Castillo et al. 1999).

It is concluded that Malvastrum yellow vein Lahore virus is a proposed new species of Begomovirus and MaYVLaV-Haz is recently identified new strain from P. vulgaris (family; Leguminosae) in northern Pakistan, whereas previously MaYVLaV-Lah was identified in M. coromandelianum from central Punjab, Pakistan.

In phylogenetic tree MaYVLaV-Lah and MaYVLaV-Haz made a separate clade between HoLCV and CLCuBaV isolates (Fig. 2A) and SDT analysis also showed maximum identity of both strains with isolates of these species. The association of CLCuMuB with MaYVLaV-Haz is not surprising as it is closely related to $\mathrm{CLCuBaV}$ that can trans-replicates betasatellites. CLCuMuB is essentially require to causing cotton leaf curl disease (CLCuD); a devastating disease of cotton in the Indian sub-continent since last three decades. This disease complex of MaYVLaV-Haz and CLCuMuB poses a real threat to other related crops and demands for its molecular characterization in other crops. In phylogenetic tree the CLCuMuB (Accession no. MK098183) isolated from green bean is closely related to CLCUMuB (Accession no. FN432359, HG428707 and KY797668 respectively; Fig. 2B). These betasatellites are isolated from Sonchus arvensis (family; Asteraceae), Gossypium hirsutum (Family; Malvaceae) and Jasminum sambac (Family; Oleaceae), respectively. The association of CLCuMuB with MaYVLaVHaz is interesting and it seems that both the components are compatible and have found a new host in Hazara district of Pakistan.

Acknowledgements We are thankful to the farmers who helped us in sampling of symptomatic green bean in Hazara district, Pakistan. We are also highly thankful to "Pakistan-U.S. Cotton Productivity Enhancement Program" of ICARDA funded by the United States Department of Agriculture (USDA), Agricultural Research Service (ARS) for funding under Agricultural Research Service (No. 58-6402$0-178 \mathrm{~F})$

Author contributions SM and IA provided the basic idea. SR wrote the manuscript. SR, MZ and NA conducted field sampling and experiment. SM, MZK, GR and IA helped in analysis and worked on the improved draft.

\section{References}

Briddon RW, Bull SE, Mansoor S, Amin I, Markham P (2002) Universal primers for the PCR-mediated amplification of DNA $\beta$. Mol Biotechnol 20:315-318

Briddon RW, Akbar F, Iqbal Z, Amrao L, Amin I, Saeed M, Mansoor S (2014) Effects of genetic changes to the begomovirus/betasatellite complex causing cotton leaf curl disease in South Asia postresistance breaking. Virus Res 186:114-119

Brown JK et al (2015) Revision of Begomovirus taxonomy based on pairwise sequence comparisons. Arch Virol 160:1593-1619

Bull S, Briddon R, Markham P (2003) Universal primers for the PCRmediated amplification of DNA 1: a satellite-like molecule associated with begomovirus-DNA $\beta$ complexes. Mol Biotechnol 23:8386

Doyle JJ, Doyle JL (1987) A rapid DNA isolation procedure for small quantities of fresh leaf tissue. Phytochem Bull 19:11-15

Muhire BM, Varsani A, Martin DP (2014) SDT: a virus classification tool based on pairwise sequence alignment and identity calculation. PloS one 9:e108277

Navas-Castillo J, Sánchez-Campos S, Díaz JA, Sáez-Alonso E, Moriones E (1999) Tomato yellow leaf curl virus-Is causes a novel disease of common bean and severe epidemics in tomato in Spain. Plant Dis 83:29-32

Shahid M, Mansoor S, Briddon R (2007) Complete nucleotide sequences of cotton leaf curl Rajasthan virus and its associated DNA $\beta$ molecule infecting tomato. Arch Virol 152:2131-2134

Tamura K, Stecher G, Peterson D, Filipski A, Kumar S (2013) MEGA6: molecular evolutionary genetics analysis version 6.0. Mol Biol Evol 30:2725-2729

Zerbini FM et al (2017) ICTV virus taxonomy profile: Geminiviridae. J Gen Virol 98:131-133 